Conference Report

\title{
The DsTau Experiment: A Study for Tau-Neutrino Production
}

\author{
Mădălina Mihaela Miloi * (D) and [DsTau Collaboration] \\ Faculty of Physics, University of Bucharest, 077125 Bucharest, Romania \\ * Correspondence: madalina.mihaela.miloi@gmail.com
}

Received: 29 December 2019; Accepted: 18 February 2020; Published: 1 March 2020

check for updates

\begin{abstract}
For clarifying the validity of the Lepton Universality hypothesis, one of the fundamental statements of the Standard Model, the interaction cross section for all three flavors of leptons have to be known with high precision. In neutrino sector, for electron and muon neutrinos, the interaction cross section is known fairly well, but for tau neutrino only poor estimations exist. In particular, the most direct measurement by the DONuT experiment was performed with rather poor accuracy due to low statistics and an uncertainty of the tau neutrino flux. The DsTau experiment proposes to study tau-neutrino production process and thus to improve significantly the accuracy of calculations of tau neutrino flux for neutrino accelerator experiments. To study reactions providing most of tau neutrinos, the experiment uses a setup based on high resolution nuclear emulsions, capable to register short lived particle decays created in proton-nucleus interactions. The present report is an overview of the DsTau experiment together with some of the preliminary results from the pilot run.
\end{abstract}

Keywords: beyond the standard model; tau-neutrino; nuclear emulsion films

\section{Introduction}

The existence of tau-neutrino was predicted in 1975 after the discovery of tau lepton [1]. In 2000, tau-neutrino charged-current (CC) interactions were observed by the DONuT experiment [2]. However, the first tau-neutrino interaction cross section measurement was performed with more than $50 \%$ systematic and 33\% statistical uncertainties, due to the uncertainty in the tau-neutrino flux and the low statistics [3].

DsTau experiment proposes to study the tau-neutrino production and to improve the calculation of tau neutrino flux in the DONuT experiment to 10\% level. Moreover, with the help of DsTau results, in future neutrino experiments like SHiP [4], where $10^{4}$ tau neutrinos interactions are expected, from about $5.7 \times 10^{15} v_{\tau}$ and $\bar{v}_{\tau}$ to be produced by $2 \times 10^{20}$ protons on target, both uncertainties in tau-neutrino interaction cross-section will be greatly reduced [5,6], making possible to the test of the Lepton Universality hypothesis.

The principal source of tau-neutrino is the leptonic decay of Ds meson [3]. The Ds to tau decay has a peculiar topology, and can be recognised in $400 \mathrm{GeV}$ proton interaction with a Tungsten target (Figure 1). There are 1000 Ds-tau events expected from $2.3 \times 10^{8}$ proton interactions, from a total initial flux of $4.6 \times 10^{9}$ protons in the DsTau experiment [5]. 


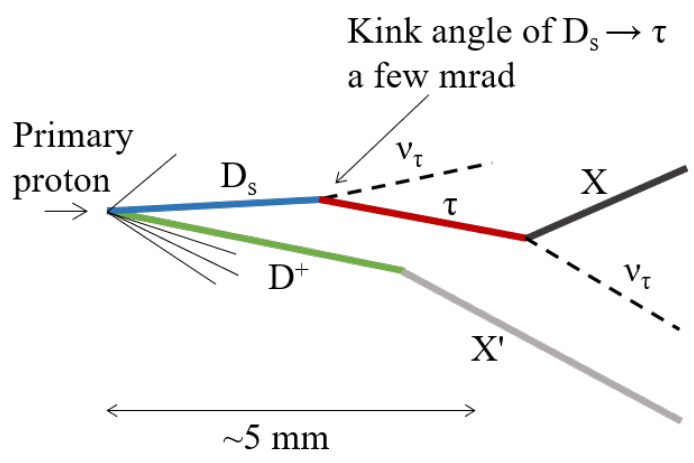

Figure 1. Ds to tau decay topology [5].

\section{Materials and Methods}

The Ds to tau events will be recognised by their double-kink topology [5], taking advantage of the best spatial resolution of the emulsion detectors. The detector module structure, as presented in Figure 2, is composed of 10 Tungsten targets. In total, 10 double-side emulsion plates on plastic support, separated by plastic plates, are inserted between two targets. At the end, an Emulsion Cloud Chamber (ECC) part is attached, composed of 26 double-side emulsion plates intercalated by Lead plates. The purpose of the ECC part is to measure the momenta of high energy particles. The proton beam is perpendicular to the detector module $[5,6]$.

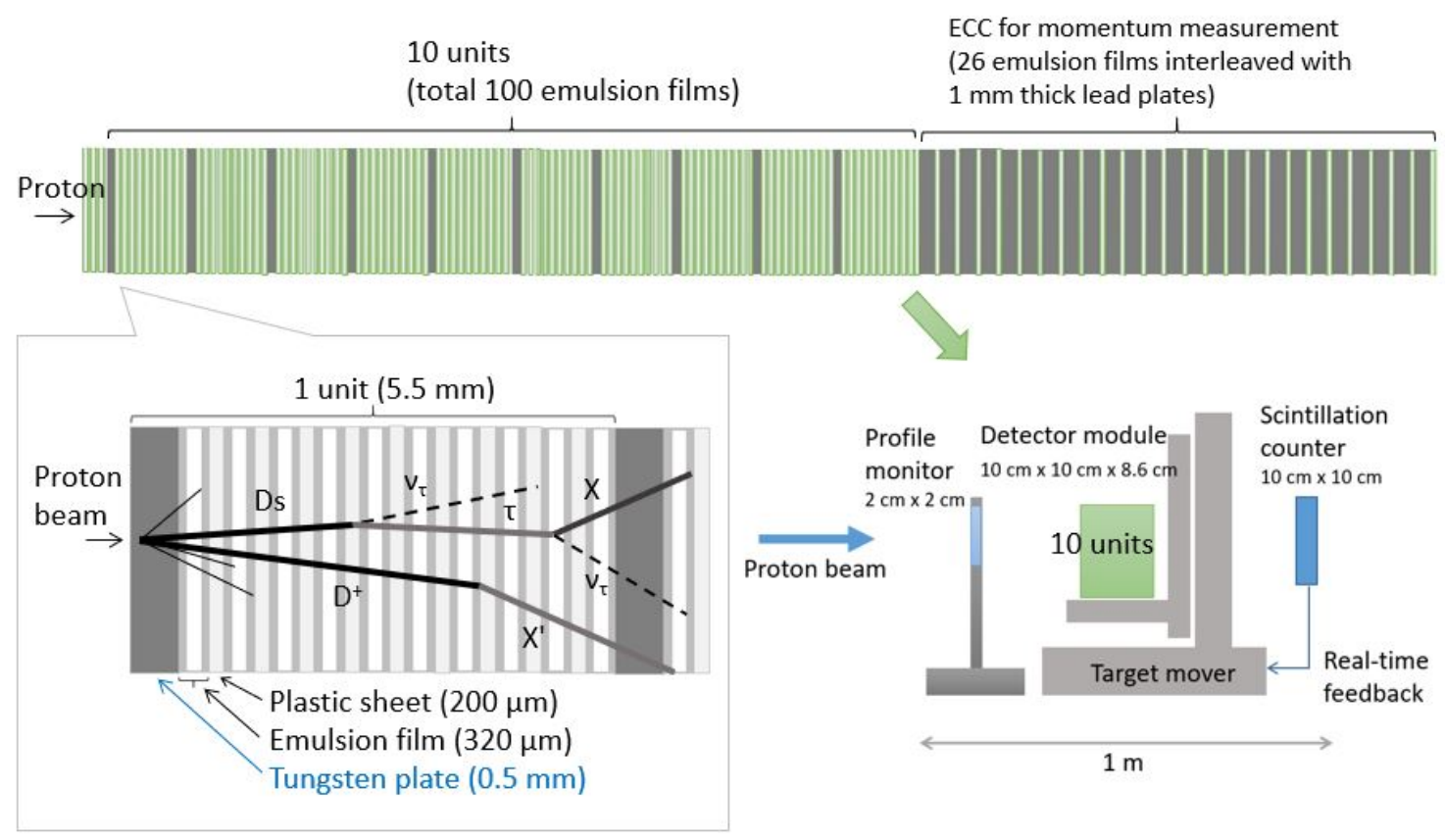

Figure 2. (Up) The detector module, consisting on 10 units (1 Tungsten +10 emulsions +9 plastic plates) and the ECC; (left) expected Ds to tau decay topology into one module; (right) the target mover together with the telescopes. This figure is form [5].

The module dimensions are $12.5 \mathrm{~cm} \times 10 \mathrm{~cm} \times 8.6 \mathrm{~cm}$. The experiment will use in total 370 modules, containing more than 48,000 emulsion plates which have to be produced before the experiment and chemically processed after the exposure. The data readout (scanning process) is automatic, using the Hyper Track Selector (HTS) which could scan a plate in $5 \mathrm{~min}$ [5]. After the scanning process, the information on all particle tracks will become available.

A module is positioned on a target mover as presented in the Figure 2, whose purpose is to change the position of the module in front of the proton beam, assuring the uniformity of the irradiation. 
Additional telescopes of pixel detectors could be added in the scope of evaluating the proton beam (Figure 2).

\section{Results}

The primary purpose of the analysis of the pilot run was to study the feasibility of the experiment. It was important to compare the experimentally observed characteristics expected from simulation. Here we present particular results for a study of multiplicity of secondary charged particles in proton interactions with different materials in detector.

The automatic data readout is followed by a geometrical reconstruction of tracks and vertexes of the proton interactions. When a vertex is found, it could be correlated with a parental proton track. In the performed preliminary analysis, only the vertexes with parent (named primary proton interactions [5]) are considered.

The vertexes distribution in the detector module is presented in Figure 3. The sub-sample of the detector structure taken into consideration is composed of one Tungsten plate and four double-side emulsion plates, together with the plastic separators and one side emulsion layer on the edges. The Tungsten plate is clearly distinguished from the other layers in terms of amount of primary proton interactions.

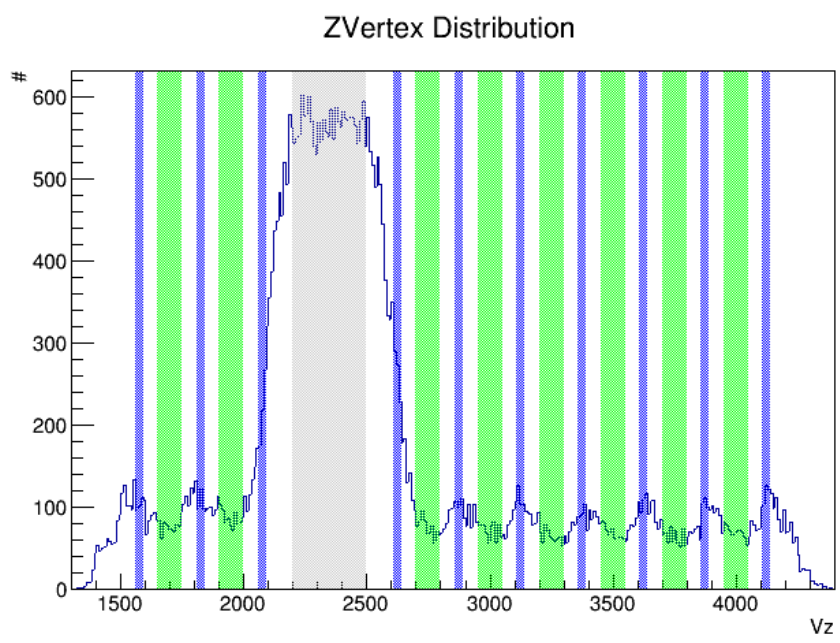

Figure 3. The distribution of the reconstructed vertexes along $\mathrm{Z}$. The structure detector is visible.

The distribution in Figure 3 is useful for distinguishing the interactions in each material. The delimitation used in the analysis is showed by colors. The grey corresponds to the cuts applied for the Tungsten plate, and the green to the cuts applied for the plastic layers. The blue corresponds to the cuts applied for the emulsion layers.

In Figure 4 the Monte Carlo (MC) comparison with the data for the multiplicity distributions in each structural material is presented. Only the charged daughters were considered from the regions indicated in the Figure 3. The MC predictions were performed by FLUKA and scaled to the number of entries in data. Further study will be done on the number of proton interactions.

In Figure 5 are presented the comparisons between the multiplicities in the three materials, for MC predictions and for the data. All the distributions were normalized to unity. 

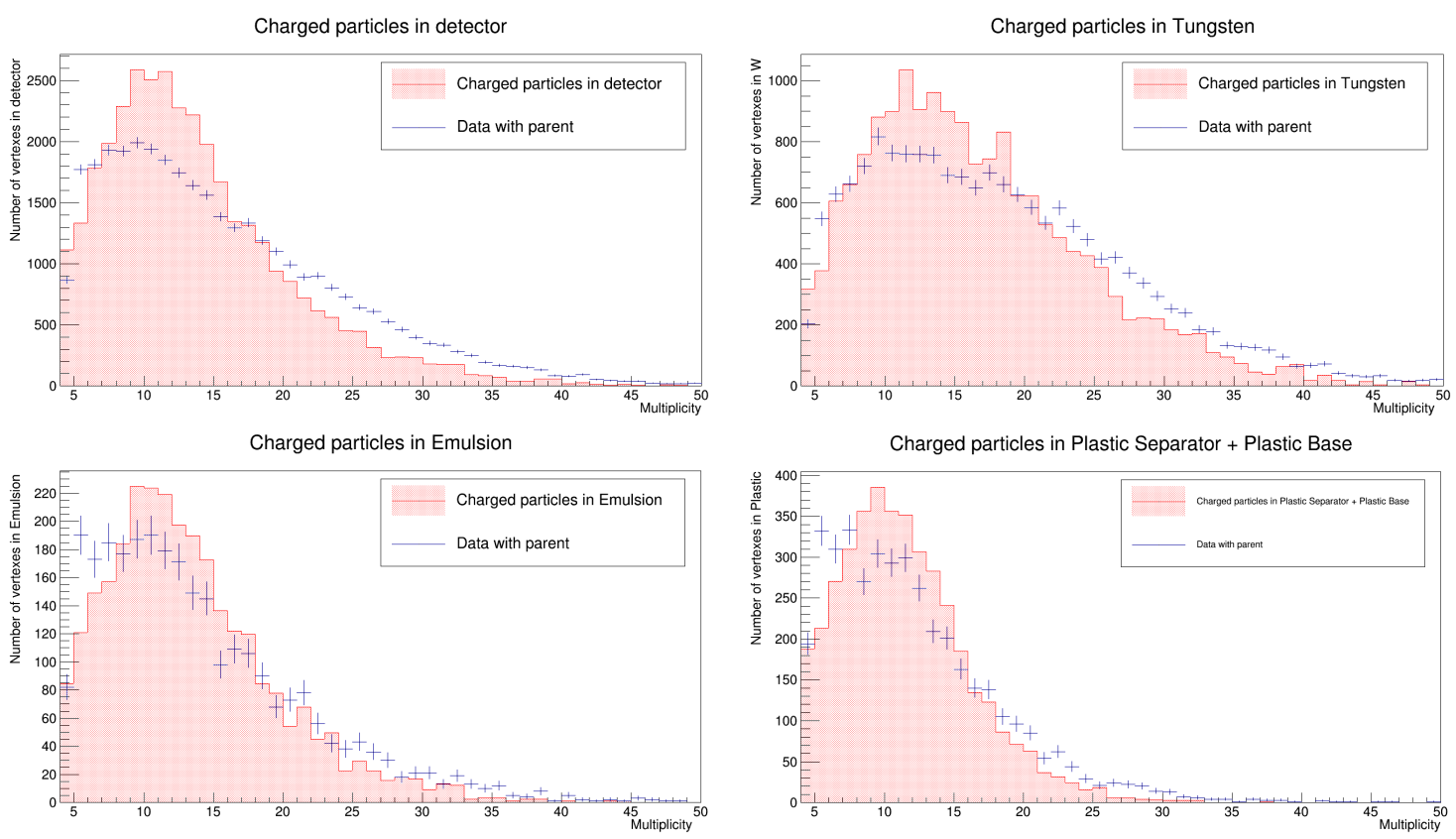

Figure 4. MC cross-checks for multiplicity distributions in each material from the detector structure.
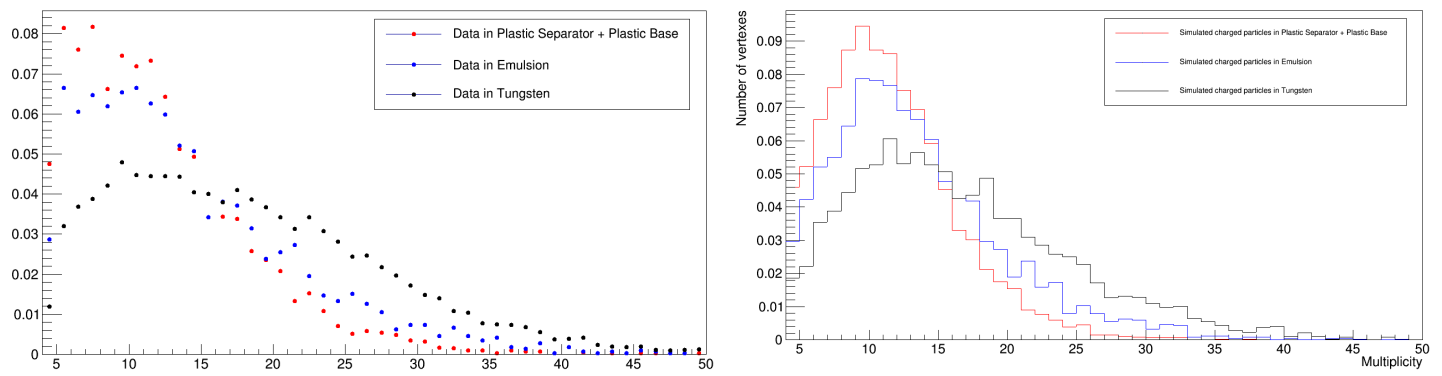

Figure 5. Comparison of normalised multiplicities for the three materials in experimental data (left) and in simulations (right).

\section{Conclusions and Outlook}

The DsTau experiment studies tau neutrino production in high energy proton interactions, which will provide the needed information for the future tau-neutrino experiments [5].

For the pilot run, the emulsion scanning as well as the analysis of the samples is an ongoing work, which will provide data for optimisation of the experiment in the main run in 2021-2022.

Funding: This research received no external funding.

Acknowledgments: First of all, I am thankful to the DsTau collaboration (without whom this work would have been impossible), for the efforts in giving me the best advice and for leading my work. Nevertheless, I want to thank to my University supervisors, Oana Ristea and Alexandru Jipa for their careful advising. Also, I want to mention Bogdan Dumitru for answering to all my questions regarding the ROOT framework.

Conflicts of Interest: The authors declare no conflict of interest.

\section{References}

1. Perl, M.L.; Abrams, G.S.; Boyarski, A.M.; Breidenbach, M.; Briggs, D.D.; Bulos, F.; Chinowsky, W.; Dakin, J.T.; Feldman, G.J.; Friedberg, C.E.; et al. Evidence for Anomalous Lepton Production in $\mathrm{e}^{+}-\mathrm{e}^{-}$Annihilation. Phys. Rev. Lett. 1975, 35, 1489. [CrossRef]

2. DONUT Collaboration; Kodama, K.; Ushida, N.; Andreopoulos, C.; Saoulidou, N.; Tzanakos, G.; Yager, P.; Baller, B.; Boehnlein, D.; Freeman, W.; et al. Observation of tau neutrino interactions. Phys. Lett. B 2001, 504, 218-224. [CrossRef] 
3. DONuT Collaboration; Kodama, K.; Ushida, N.; Andreopoulos, C.; Saoulidou, N.; Tzanakos, G.; Yager, P.; Baller, B.; Boehnlein, D.; Freeman, W.; et al. Final tau-neutrino results from DONuT experiment. Phys. Rev. D 2008, 78, 052002. [CrossRef]

4. Anelli, M.; Aoki, S.; Arduni, G. A facility to Search for Hidden Particles (SHiP) at the CERN SPS, CERN-SPSC-2015-016, SPSC-P-350. arXiv 2015, arXiv:1504.04956.

5. DsTau Collaboration; Aoki, S.; Ariga, A.; Ariga, T.; Dmitrievsky, S.; Firu, E.; Forshaw, D.; Fukuda, T.; Gornushkin, Y.; Guler, A.M.; et al. DsTau: Study of tau neutrino production with $400 \mathrm{GeV}$ protons from the CERN-SPS. J. High Energy Phys. 2020, 2020, 1-26.

6. Aoki, S.; Ariga, A.; Ariga, T.; Firu, E.; Fukuda, T.; Gornushkin, Y.; Guler, A.M.; Haiduc, M.; Kodama, K.; Korkmaz, M.A.; et al. Experiment Proposal, Study of tau-neutrino production at the CERN SPS, CERN-SPSC-2017-029, SPSC-P-354. arXiv 2017, arXiv:1708.08700.

(C) 2020 by the authors. Licensee MDPI, Basel, Switzerland. This article is an open access article distributed under the terms and conditions of the Creative Commons Attribution (CC BY) license (http:// creativecommons.org/licenses/by/4.0/). 